\section{La variable ambiental en el eje relacional centro-periferia según la perspectiva teórica Análisis de Sistema-Mundo. Un análisis en los roles de América Latina en su relación con los países centrales del sistema mundial ${ }^{1}$}

A variável ambiental no eixo da relação centro-periferia segundo a perspectiva teórica da Análise do SistemaMundo. Uma análise dos papéis da América Latina em sua relação com os países centrais do sistema mundial.

The Environmental Variable In The Core-Periphery Relational Axis According To The World-System Theoretical Perspective. An Analysis On Latin American Roles In Its Relation With The Core Of The World-Systems

Juan Pablo Vásquez Bustamante ${ }^{3}$

DOI: 10.5752/P.2317-773X.2019v7.n2.p101

Recibido el 06 de julio de 2018

Aprobado el 06 de noviembre de 2018

\section{RESUMEN}

Este artículo propone incorporar la crisis ambiental como variable en el eje relacional centro-periferia según la perspectiva teórica Análisis de Sistema-Mundo, en la búsqueda de identificar posibles cambios en sus flujos y en los roles de América Latina como región periférica. A través de un análisis bibliográfico, se buscan las principales referencias planteadas en la perspectiva teórica Análisis de Sistema-Mundo respecto al fenómeno de la crisis ambiental; se identifican y describen los principales elementos que definen el eje relacional centro-periferia según la perspectiva teórica Análisis de Sistema-Mundo; se identifican y describen algunos efectos y consecuencias de la crisis ambiental planteados por intelectuales latinoamericanos vinculados a la Comisión Económica para América Latina y el Caribe (CEPAL), que pudiesen constituirse en factores de cambio en las relaciones entre centro y periferia, y se intenta establecer una asociación entre ellos y determinados antecedentes, elementos y problemáticas a partir del caso de la propuesta ecuatoriana denominada Iniciativa Yasuní ITT.

Palabras Clave: Análisis de Sistema-Mundo. Crisis ambiental. Centro-Periferia. América Latina.
1. Este artículo es producto de la investigación de tesis doctoral: "La variable ambiental en el eje relacional centro-periferia según la perspectiva teórica Del Sistema Mundo. Un análisis sobre la base de los estudios de los casos de Ecuador y Bolivia entre los años 2005 y 2015". Doctorado en Estudios Americanos, Universidad de Santiago de Chile.

El autor agradece al Vicedecanato de Investigación y Postgrado de la Facultad de Humanidades y a la Vicerrectoría de Postgrado de la Universidad de Santiago de Chile el apoyo otorgado para la realización de esta investigación y el desarrollo de este artículo.

2. Traducido por la Traductora Inglés-Español y Licenciada en Lenguas y Letras Constanza Morales Peñaloza

morales.constanza.alice@gmail.com

3. Candidato a Doctor en Estudios Americanos, especialidad Estudios Internacionales, Instituto de Estudios Avanzados, Universidad de Santiago de Chile. Magíster en Estudios Internacionales, Universidad de Santiago de Chile. Coinvestigador proyecto Fondecyt $\mathrm{N}^{\circ}$ 1190481. Profesor en la línea de Historia en la Universidad Miguel de Cervantes. Santiago, Chile. Correo electrónico: juan.vasquez@usach.cl ORCID: https:// orcid.org/0000-0001- (iD) 


\section{RESUMO}

Este artigo propõe a incorporação da crise ambiental como uma variável no eixo relação centro-periferia de acordo com a perspectiva teórica de Análise do Sistema-Mundo, buscando identificar possíveis mudanças nos fluxos e no papel da América Latina como região periférica. Por meio de uma análise bibliográfica, buscam-se as principais referências propostas na perspectiva teórica da Análise do Sistema-Mundo sobre o fenômeno da crise ambiental; identificam-se e descrevem-se os principais elementos que definem o eixo da relação centro-periferia de acordo com a perspectiva teórica de Análise do Sistema-Mundo; além de alguns efeitos e consequências da crise ambiental representada pelos intelectuais latino-americanos ligados à Comissão Económica das Nações Unidas para a América Latina e o Caribe (CEPAL), podem se tornar agentes de mudança nas relações entre o centro e a periferia, e por fim, busca-se estabelecer um vínculo entre eles e determinados antecedentes, elementos e problemas a partir da proposta equatoriana denominada Iniciativa Yasuni ITT.

Palavras-chave: Análise do Sistema-Mundo. Crise ambiental Centro-periferia. América Latina.

\section{Abstract}

This article aims to add the Environmental Crisis as a variable in the core-periphery relational axis according to the theoretical perspective of the World-Systems Analysis in the search of identifying potential changes in its flows and in Latin American roles as a peripheral region. Throughout a literature review, the main references of the World-Systems Analysis theoretical perspective regarding the Environmental Crisis phenomenon are searched for; the main defining aspects of the core-periphery relational axis according to the World-System Analysis are identified and described; some effects and consequences of the Environmental Crisis - which are lay out by Latin-American intellectuals linked to the ECLAC - that are potential change factors between core and periphery are set out: in addition, a relation between those change factors and determined elements and problematic precedents of the Ecuadorian Yasuní ITT Initiative -a global environmental proposal- is offered.

Key words: World-Systems Analysis. Environmental Crisis. Core/Periphery. Latin America.

Introducción

Se entiende por perspectiva teórica Análisis de Sistema-Mundo (AS-M), un enfoque analítico que reúne un conjunto de teorizaciones, hipótesis y modelos explicativos respecto al desarrollo histórico global del capitalismo y la modernidad (AGUIRRE, 2004, p. 34).

Bajo este enfoque, los sistemas-mundiales corresponden a zonas espacio temporales que atraviesan múltiples unidades políticas y culturales, integradas de actividades e instituciones que obedecen a ciertas reglas sistémicas. Es factible identificar dos tipos de sistemas mundiales, los imperios-mundo y las economías-mundo (WALLERSTEIN, 2005, p. 33).

El moderno sistema-mundo, correspondiente a una economíamundo originada entre fines del siglo XV y principios del XVI y actualmente en proceso, está concebido como una unidad expansiva, primero semiplanetaria, y posteriormente planetaria, cuya curva evolutiva es la historia específica de la génesis, evolución y crisis sistémica del capitalismo 
como sistema histórico global (AGUIRRE, 2004, p. 34-35).

Dentro de la variedad de premisas, modelos e hipótesis que entrega este marco analítico, dos de ellas son particularmente importantes para esta investigación. En primer lugar, la unidad de análisis fundamental no es ni el Estado soberano ni la sociedad nacional. La unidad correcta es el propio sistema mundial. Son los procesos en desarrollo, generados a escala planetaria, los que proporcionan el verdadero objeto de la investigación colectiva (AGUIRRE, 2004, p. 38; WALLERSTEIN, 2007a, p. 85; WALLERSTEIN, 2010, p. 12).

En segundo lugar, el moderno sistema-mundo, la economía-mundo capitalista, manifiesta un orden y una división del trabajo expresada en un conjunto de relaciones e intercambios asimétricos y desiguales, donde sus partes se dividen en zonas centrales, áreas periféricas, un bloque intermedio denominado semiperiferia y "arenas externas", es decir, zonas que están afuera de las fronteras del sistema, y que, potencialmente, a partir de la naturaleza expansiva del mismo, podrían ser incorporadas (AGUIRRE, 2004, p. 41-45).

Este eje relacional centro-periferia, tomado del esquema conceptual propuesto por el economista argentino Raúl Prebisch, tiene como característica decisiva un flujo asimétrico de plusvalía, es decir, tasas de excedentes que se van hacia los Estados centrales, a partir, entre otras cosas, de actividades productivas con mayor valor (AGUIRRE, 2004, p. 225-226). Estas unidades presentan, además, una mayor capacidad de generar una institucionalidad estable y soberana, a diferencia de los Estados periféricos que se ven desfavorecidos por esta relación.

A partir de estas dos propuestas, se entiende que los fenómenos problemáticos observados bajo el enfoque de la perspectiva teórica AS-M, son tensiones de naturaleza global que responden a corrientes de escala mundial.

Por otro lado, uno de los fenómenos problemáticos de la realidad global contemporánea, que precisamente opera en una escala mundial, es la denominada Crisis Ambiental (CA), la cual, para el Dr. Fernando Estenssoro (2007, p. 94), se entiende como el paradójico fenómeno de que tanto el desarrollo, los patrones y el estándar de vida alcanzados por la "Civilización Industrial", en la cual, su arquetipo son los países desarrollados del Primer Mundo, han creado problemas de carácter ecológico y medioambientales de tan enorme magnitud, que han puesto en riesgo la continuidad de la vida del ser humano en el planeta, así como la vida del planeta mismo ${ }^{4}$.

A partir del reconocimiento de la CA como una realidad problemática de la sociedad global actual, y de la responsabilidad y necesidad de encontrar respuestas ante ella, es que se ha generado una "intensa polémica política e ideológica" denominada debate ambiental (ESTENSSORO, 2009, p. 11). El cual, se manifiesta como un debate político, desarrollado a través de relaciones de poder, donde los diferentes actores se enfrentan con apreciaciones diversas, confrontadas, difíciles de conciliar, derivadas de sus necesidades, intereses, marcos ideológicos y capacidad de influir en el otro (ESTENSSORO, 2014).

Este debate ambiental, durante las últimas décadas del siglo XX y los primeros decenios de la presente centuria, se ha constituido en uno de
4. La crisis ambiental es un fenómeno complejo, cuyos elementos causales, efectos y consecuencias operan y se hacen perceptibles en diferentes escalas, tanto a nivel global como local. En consecuencia, las diversas miradas y estudios en el marco del debate ambiental la abordan desde diferentes ángulos, y, por cierto, con distintos grados de abstracción, de acuerdo al nivel del cual se esté tratando. Este trabajo aborda la crisis ambiental en su dimensión global y en su relación con el desarrollo de la economía-mundo capitalista, desde una perspectiva que se plantea con un enfoque histórico y sistémico. 
los principales ítem tanto de la agenda política de los actores del Sistema Internacional, compuesto por Estados Nacionales y Organizaciones Internacionales, como de las preocupaciones de la Sociedad Internacional, entidad que involucra tanto el accionar de los Estados, como también de individuos y organismos (ESTENSSORO, 2007; ORTIZ, 2011, p. 19).

No obstante la relevancia mundial que ha tomado el fenómeno problemático de la CA y la discusión en torno suyo, el debate ambiental, pese a la presumible relación que podrían tener el desarrollo del capitalismo histórico y un fenómeno provocado justamente por sus procesos productivos, por los patrones de vida de la sociedad industrial, por el consumo masivo, por la racionalidad imperante en sus lógicas, a pesar de constituirse en un enfoque analítico sistémico, histórico y totalizante, la perspectiva teórica AS-M parece no referirse a esta problemática en sí misma, aparentemente pareciera no existir una vinculación epistemológica estrecha, directa, explícita e intencionalmente abierta.

Este trabajo buscar incorporar la CA como variable en el eje relacional centro-periferia según la perspectiva teórica AS-M, en la búsqueda de posibles cambios en sus flujos y roles, especialmente en la periferia, y dentro de ella en América Latina. Se pretende, de una parte, establecer una mirada analítica que pudiese enriquecer la perspectiva teórica AS-M, $y$, por otro lado, identificar elementos que se constituyan en antecedentes y herramientas para una mayor comprensión de los roles, la posición y las oportunidades de la periferia, y especialmente de los países latinoamericanos, en el sistema mundial y en la actual fase del desarrollo del capitalismo histórico.

En las líneas a continuación: en primer lugar, se identifican y describen menciones, referencias y vínculos planteados en la perspectiva teórica AS-M respecto al fenómeno de la CA; en segundo lugar, se identifican y describen los principales elementos que definen el eje relacional centroperiferia según la perspectiva teórica AS-M; en tercer lugar, se identifican y describen algunos efectos y consecuencias de la CA, planteados al inicio de la década de los ochenta por intelectuales latinoamericanos vinculados a la Comisión Económica para América Latina y el Caribe (CEPAL), que pudiesen constituirse en factores de cambio en las relaciones entre centro y periferia, y se intenta establecer una asociación entre ellos y determinados antecedentes, elementos y problemáticas a partir del caso de la propuesta ecuatoriana denominada Iniciativa Yasuní ITT; finalmente, se ofrecen algunas conclusiones y reflexiones al cierre.

Perspectiva teórica Análisis de Sistema-Mundo y Crisis Ambiental:

Transformación/depredación ecológica, origen, expansión y crisis estructural de la economía-mundo capitalista

La vinculación entre la CA y la perspectiva teórica AS-M parece ser un ejercicio inferencial a desarrollar, pues la obra que se constituye en la columna vertebral de este enfoque teórico, los cuatro tomos del Moderno Sistema Mundial de Immanuel Wallerstein (1974; 1980; 1989; 2011) no habrían planteado explícitamente ni desarrollado en sí misma esta problemática contemporánea y global. Sin embargo, al revisar y analizar con 
atención esta obra y posteriores publicaciones que la complementan y que están bajo esta perspectiva teórica, es posible encontrar a lo menos dos puertas comunicantes.

En primer lugar, para Immanuel Wallerstein (2010, p. 53-54) existen tres elementos fundamentales para el establecimiento de la economía-mundo capitalista: la expansión del volumen geográfico del "mundo en cuestión", el desarrollo de variados métodos de control del trabajo para diferentes productos y zonas de la economía-mundo, y el surgimiento de Estados fuertes que posteriormente se transformarían en los Estados centrales del sistema mundial. Más aún, el segundo y tercero de estos factores dependían del primero.

Ese primer elemento, la expansión territorial europea, fundamental en la superación de la crisis del Feudalismo y en el surgimiento y desarrollo del capitalismo histórico, se conecta con la CA a través de dos caminos. Por una parte, el ensanchamiento geográfico de la economía-mundo iba dejando a su paso una alteración de la ecología mundial (WALLERSTEIN, 2010, p. 63), a partir, principalmente, de la introducción de sus procesos productivos, los cuales implicaban, entre otras cosas, la utilización progresiva e intensiva de los suelos y la extracción y usos de elementos de la naturaleza a ritmos hasta entonces no experimentados en aquellas zonas recientemente incorporadas a la economía-mundo.

Por otro lado, es preciso preguntarse por las motivaciones iniciales que llevaron a aquella expansión geográfica de Europa Occidental, tan esencial para el surgimiento y desarrollo de la economía-mundo capitalista. Para Wallerstein (2010, p. 69), lo que incitó y sostuvo esta empresa fue la necesidad de comida y combustible, pues Europa Occidental necesitaba más calorías y una mejor distribución de los valores alimenticios. Ante este escenario, ¿por qué no incrementar la producción dentro de sus propias fronteras? Pues bien, aquello se realizó en determinadas regiones sobre la base de mejoras tecnológicas, sin embargo, estos casos se dieron precisamente en lugares con una base demográfica densa y con un proceso de crecimiento industrial, lo cual, de todas formas, requirió la importación de grandes cantidades de granos. ¿Y por qué no, entonces, ensanchar las fronteras agrícolas dentro de los límites de la propia Europa? Aquello también se realizó, sin embargo, el espacio fue insuficiente (WALLERSTEIN, 2010, p. 61). Ante lo cual, el camino seguido fue la expansión geográfica de la Europa Occidental.

En esta dinámica el caso del azúcar es particularmente interesante, útil como fuente de calorías, como sustitutivo de las grasas, como materia prima para licores e ingrediente en la preparación del chocolate, este alimento se constituyó en una de las principales motivaciones para la expansión de Portugal por el Atlántico (WALLERSTEIN, 2010, p. 62). Se trataba de un producto lucrativo que expulsaba al trigo y que gastaba el suelo, lo que, lejos de ser un problema, en el largo plazo motorizaba la economía-mundo capitalista, pues "requería continuamente nuevas tierras", es decir: expansión territorial (WALLERSTEIN, 2010).

Además de alimentos y calorías, Europa Occidental necesitaba madera para leña y construcciones. Situación ante lo cual se recurría a las importaciones, y que, al igual que el azúcar, también motivó el proceso de 
5. Respecto a esta relación causal entre capitalismo y Crisis Ambiental, existen diversos intelectuales y líneas de pensamiento que profundizan sobre ella y la establecen en el centro de su análisis. En este marco pueden plantearse la obra de Jason Moore (2003a; 2003b; 2013) y el enfoque denominado Worl$d$-Ecology, que en varias de sus líneas fundamentales se desprende de la perspectiva teórica AS-M. Así mismo, puede considerarse el trabajo de John Bellamy Foster (1999) y, entre otros aportes, sus planteamientos en torno al metabolic rift. De igual forma, en este contexto puede mencionarse la corriente de pensamiento Ecosocialismo, la cual critica el modo de producción y consumo capitalista planteándolo incompatible con la ecología y la protección de la naturaleza. El Ecosocialismo se hace

parte de la tradición socialista e integra ciertos elementos fundamentales del marxismo con la ecología política, constituyéndose en una corriente de "pensamiento y acción ecológica", de la cual son parte intelectuales como Michael Löwy, Jorge Riechmann, James O'Connor, entre otros (LE QUANG; VERCOUTERE, 2015). expansión territorial hacia fuera de sus fronteras en la búsqueda de estos insumos, pues: "el desarrollo económico de la Edad Media, y se debe asumir que también sus crudas técnicas forestales, habían llevado a una lenta pero constante deforestación de Europa Occidental, Italia, España, así como también de las Islas Mediterráneas" (WALLERSTEIN, 2010, p. 63).

Es decir, la expansión geográfica de la Europa Occidental de los siglos XV y XVI, esencial para el surgimiento y desarrollo de la economíamundo capitalista, fue generando a su paso una transformación/depredación ecológica progresiva en la medida en que aquella expansión también lo fuese. Y a su vez, una de las motivaciones que empujó aquella expansión territorial europea que transformaba/depredaba la ecología mundial, era encontrar nuevos suelos para la agricultura y nuevas fuentes de recursos, pues dentro de sus fronteras ya se habían utilizado y/o depredado.

Es decir, siguiendo la perspectiva teórica AS-M, puede afirmarse que existe una correlación directa y positiva entre surgimiento, desarrollo y expansión de la economía-mundo capitalista y la transformación/ depredación ecológica, y a la larga la CA. Pues, la expansión territorial, esencial y clave en el origen y desarrollo del capitalismo histórico, provoca transformación y depredación ambiental, y a su vez, a lo menos en parte, es provocada, motivada y motorizada por ella. Como plantea el historiador Jason Moore (2013a), las "transformaciones de la tierra" fueron a la vez consecuencia y constituyentes del auge del capitalismo. Bajo su interpretación, la expansión europea posterior a 1492 fue parte de un cambio de época en las relaciones entre naturaleza y sociedad, el cual era a la vez causa y consecuencia del auge del capitalismo (MOORE, 2013a, p. 23).

Si bien, las expansiones, el colonialismo, el rompimiento de fronteras, eran fenómenos que se experimentaban desde hacía siglo atrás, la "geografía-histórica del colonialismo" en su forma moderna, posterior a 1492, "debe ser comprendida dentro de la incipiente dialéctica de la acumulación por apropiación y la acumulación por capitalización”, donde la producción y el intercambio de mercancías pasaron a constituirse en fines en sí mismos (MOORE, 2013a, p. 23).

En este sentido, y complementando críticamente la conceptualización del Dr. Estenssoro (2007), podría afirmarse que la CA, si bien explota notoriamente con los patrones de vida de la Sociedad Industrial, es esencialmente producto e hija del desarrollo del capitalismo histórico desde sus orígenes, allí están sus causas, e inclusive allí pueden percibirse algunos de sus efectos notorios.

Para Moore, a diferencia de la "vasta historiografía" y los largos debates en torno a la transición del feudalismo al capitalismo, Wallerstein efectivamente vio la crisis feudal como una "coyuntura socio-física" y el auge del capitalismo como una reorganización de la ecología mundial (MOORE, 2003a; 2013b, p. 16) .

La segunda puerta comunicante entre la perspectiva teórica AS-M y la CA, abierta por el propio Wallerstein (2007b), tiene que ver con la hipótesis de que la economía-mundo capitalista habría entrado a una crisis terminal a partir del desarrollo de tres fallas estructurales en su capacidad de acumulación de capital. Para este científico social norteamericano: "Como la acumulación sin límite de capital es el rasgo definitorio del ca- 
pitalismo como sistema histórico, la triple presión tiende a volver inviable el motor primario del sistema y por lo tanto crea una crisis estructural" (WALLERSTEIN, 2007b, p. 54).

La primera de estas fallas es el incremento del costo del trabajo como porcentaje del total del valor creado, producto de la desruralización del mundo. Pues, este fenómeno limita para el capital la posibilidad de reubicar sectores de la producción en zonas de más bajos salarios y reclutar a migrantes rurales que recientemente se incorporen al mercado del trabajo asalariado. Mecanismo con el cual tradicionalmente los capitalistas abaratan los costos, incrementan sus excedentes y limitan las potenciales presiones de las organizaciones sindicales (WALLERSTEIN, 2007b).

La segunda de estas tres fallas estructurales planteadas por Wallerstein (2007b, p. 228), tiene directa relación con la CA. Se trata de los límites ecológicos a la contaminación y la no renovación de los recursos, lo que limita la capacidad del capital para reducir los costos de los insumos por la vía de su externalización continua.

Para Wallerstein (2001a; 2007b, p 209), el principal mecanismo de los capitalistas para mantener bajos los costos de los insumos ha sido externalizarlos, es decir: no pagar algunos de ellos consiguiéndolos a costa de la sociedad. Esto se ha logrado, principalmente, a través de tres formas: eliminar los desechos del material, que pudiesen ser tóxicos, al exterior de la propiedad de la industria o empresa arrojándolos a un espacio natural común; comprar y utilizar materias primas sin asumir los costos de su renovación o de su sustentabilidad, por ejemplo, deforestar sin hacerse responsable por la posterior reforestación; y emplear infraestructura a costa de la sociedad.

En los dos primeros casos, se hace necesaria la localización de áreas donde, por diversos motivos, pueda arrojarse el desecho del material, y zonas donde conseguir materias primas a un bajo costo y sin la necesidad de invertir en procesos sustentables. Con la incorporación permanente de áreas dentro de la economía-mundo capitalista y la transformación/ depredación ecológica en distintas zonas producto de los procesos de producción, el planeta se va quedando sin lugares de reemplazo. En la tercera de las formas de externalización de costos, para el empleo de infraestructura construida a expensas de la sociedad, se requiere un aumento constante de los impuestos, situación que se articula con la tercera de las fallas estructurales (WALLERSTEIN, 2007b, p. 209-210).

Pues, para Wallerstein (2007b, p. 58-59), la tercera de estas fallas sería producto de las respuestas de los gobiernos a las presiones y demandas populares por mayores derechos y bienestar social, lo cual requiere permanente financiamiento y por lo tanto ha implicado el constante incremento de las tasas de tributación. Este sistema de redistribución fiscal empuja los costos de los impuestos como porcentaje del valor del total creado, hasta un punto en que limita la capacidad de acumulación de capital, lo cual genera toda una dinámica de relaciones políticas complejas a partir de la posible reducción de prestaciones sociales versus el incremento de las cargas fiscales. En ambos casos, tercera falla estructural y tercera forma de externalización de costos de la segunda contradicción anteriormente expuesta, la solución sería que el capital asumiera el costo a través de su carga tributaria, lo que limitaría su capacidad de acumulación. 
Para Wallerstein (2007b, p. 207), la economía-mundo capitalista ha llegado a un punto de bifurcación en la cual no es capaz de superar determinadas contradicciones, las cuales, en este caso, tienen que ver con que a lo largo de más de cinco siglos de funcionamiento, los costos del trabajo, la inversión e infraestructura y la carga tributaria han ido aumentando constantemente como un porcentaje del valor total de lo producido, con el resultado de entrar en una fase en la cual se experimenta una reducción de las utilidades a nivel global que amenaza el funcionamiento básico y esencial del sistema: su capacidad de acumulación incesante de capital, significando un proceso de crisis estructural y terminal de la economia-mundo capitalista, y siendo dentro suyo el agotamiento ecológico uno de los factores fundamentales.

De este modo, la vinculación entre la CA y la perspectiva teórica AS-M se convierte en un ejercicio académico y cognitivo paradójico, donde, por una parte, hay dos puertas comunicantes claves prácticamente en los extremos: podría decirse que la transformación/depredación de los ecosistemas es esencial como motor y consecuencia en el surgimiento y desarrollo de la economía-mundo capitalista, al mismo tiempo que el agotamiento ecológico sería uno de los factores de determinadas fallas estructurales conducentes a una crisis final. Mientras, pese a aquello, esta perspectiva teórica ha sido mayoritaria y tradicionalmente entendida bajo un lente de "exencionalismo humano" (MOORE, 2003a, p. 308).

Si efectivamente la transformación/depredación ecológica fue fundamental para el surgimiento y desarrollo de la economía-mundo capitalista, y si además la CA, que es consecuencia directa de esa transformación/depredación ecológica, es un factor esencial de una de sus fallas estructurales conducente a una crisis terminal, y tomando una de las preguntas más agudas y críticas de Wallerstein (2001, p. 89) respecto a la CA, ¿para quién es el peligro? ¿A quién afecta principalmente este fenómeno problemático?, es preciso preguntarse: ¿Qué incidencia tiene la incorporación de la CA como variable en el eje relacional centro-periferia según la perspectiva teórica AS-M? ¿Genera la CA algún tipo de modificación en el eje relacional centro-periferia? ¿Se puede considerar a la CA como una variable de cambio en ese conjunto de relaciones? ¿Tal vez favorable a la periferia? ¿Tal vez incrementadora de la asimetría a favor del centro?

El eje relacional centro-periferia según la perspectiva teórica Análisis de Sistema-Mundo

Para Wallerstein (2010), un sistema mundial es un sistema social. El cual posee límites, reglas, partes que lo componen, organismos que habitan y cohabitan en su interior, un funcionamiento coherente que permite su subsistencia.

Lo que caracteriza a un sistema social, es el hecho de que la vida en su seno está en gran medida autoincluida, y que la dinámica de su desarrollo es en gran parte interna. Si el sistema, por cualquier razón, quedara aislado de todas las fuerzas externas, por definición, incluso, continuaría funcionando sustancialmente de la misma manera (WALLERSTEIN, 2010).

Wallerstein (2007a, p. 249) concibe este sistema social como una sucesión y coexistencia de múltiples entidades de largo plazo y de gran 
escala, a los que denomina como sistemas históricos. Los cuales presentan tres características que los definen: primero, relativa autonomía, es decir que funcionan a partir de sus propios procesos internos, sus fuerzas motrices son endógenas; en segundo lugar, tienen límites temporales, necesariamente nacen y posteriormente mueren en tiempos determinados; finalmente, tienen límites espaciales, aunque esas fronteras pueden ir experimentando modificaciones a lo largo de su existencia.

Un sistema histórico representa una red integrada de procesos económicos, políticos y culturales que lo mantienen unido. En tanto sistema, si cualquier proceso particular de la red cambia alguno de sus parámetros, los otros, de cualquier manera, deberán adaptarse. Dentro de un sistema histórico en funcionamiento no existe libre albedrío, son las estructuras de este sistema quienes crean, limitan y ofrecen las distintas opciones (WALLERSTEIN, 2007a, p. 249).

Un sistema es una entidad real, el cual presenta un cierto contenido, y donde todas sus partes son interdependientes entre sí. Si desaparece una de esas partes, el sistema por completo se ve alterado (WALLERSTEIN apud AGUIRRE 2004, p. 229). Bajo esta lógica, y dentro de la concepción del funcionamiento de un sistema, se entiende sistema-mundo en consecuencia, el moderno sistema-mundo, y por tanto la economía-mundo capitalista, nuestro sistema global actual, como un entramado de organismos y estructuras vinculadas relacionalmente entre sí. Así como el sistema seguiría funcionando sustancialmente de la misma manera si quedara aislado de las fuerzas externas, al contrario, cualquiera de las partes del sistema dejaría de funcionar si quedara aislada como unidad autónoma respecto del funcionamiento del mismo. Cada una de las unidades en sí misma carece de la capacidad de generar un funcionamiento diferente y propio respecto del funcionamiento sistemático.

A partir de esta propuesta teórica, es que la unidad de análisis es justamente la totalidad, el propio sistema-mundo, no las partes, no los estados y/o las sociedades nacionales. Estos no poseen ni la autonomía ni el aislamiento que hiciese posible calificarlos como poseedores de un modo particular de relaciones de producción (WALLERSTEIN, 2005, p. 37). Desde una perspectiva menos abstracta y más teórico política, significa que ningún Estado ha sido realmente soberano dentro de sus fronteras, así mismo que nunca un Estado lo ha sido verdaderamente hacia afuera (WALLERSTEIN, 2001b, p. 70).

Asumiendo como premisas relacionadas y dialógicas los elementos teóricos anteriores, es preciso entender que aquella interrelación entre las partes del sistema del que acá se está tratando, no es ni equilibrada ni simétrica. Es decir, el moderno sistema-mundo, o sea, la economíamundo capitalista está estructurada por una división global del trabajo, a partir de un eje relacional del que son parte: zonas caracterizadas por sistemas productivos centrales; y zonas caracterizadas por sistemas productivos periféricos. Entre ellas se establece una estructura intermedia denominada semiperiferia (WALLERSTEIN, 2010). Y, finalmente, por fuera de los límites de la periferia, se pueden considerar las denominadas "arenas exteriores" (AGUIRRE, 2004, p. 41 - 45).

Para Wallerstein (2005, p. 34), el eje centro-periferia se constituye en un concepto relacional, es decir, cada uno adquiere su sentido en el 
clivaje a partir de la propia relación con el otro. Este orden implica una jerarquización en la organización del trabajo, los roles que requieren una mayor cualificación y capacitación son parte de las tareas de los Estados centrales, quienes, en tanto, acumulan más capital, viéndose favorecidos con la mayor parte del excedente del trabajo (WALLERSTEIN, 2010).

Para Wallerstein (2005, p. 34), el resultado de esta relación de roles e intercambios desiguales entre áreas periféricas y áreas centrales, genera como resultado un flujo de plusvalía, una gran cantidad de las ganancias y excedentes de producciones locales se trasladan desde las zonas periféricas hacia los Estados del centro.

Esta organización del trabajo y de las relaciones de producción es funcionalmente complementada con una dinámica desarrollada al interior de la economía-mundo, donde los Estados del centro presentan estructuras relativamente fuertes, mientras los Estados de las áreas periféricas manifiestan desde una relativa debilidad a una debilidad estructural, que oscila entre la no existencia del Estado y la existencia con escaso grado de autonomía (WALLERSTEIN, 2010, p. 499).

Con estructuras estatales fuertes, el autor se refiere a su fuerza en relación a otros Estados del centro y la periferia, a unidades políticas locales y a cualquier grupo social particular dentro de un Estado. Ningún Estado es totalmente soberano, ni interna ni externamente, sin embargo, un Estado fuerte sería aquel con la suficiente autonomía parcial, que le entregue un margen de acción frente a múltiples intereses de distintos grupos de fuerza. Por el contrario, en un Estado débil sus administradores carecen de la capacidad de coordinar los procesos internos, se convierten "simplemente en un grupo de terratenientes entre los otros, con poco derecho a afirmar su autoridad sobre la totalidad" (WALLERSTEIN, 2010, p. 500-501).

La estructura estatal, tanto para el centro y la periferia, supone un mecanismo basculante, es decir, existe un punto en que la fuerza es creativa de más fuerza, al igual que la debilidad se convierte en generadora de más debilidad. Esta proposición tiene expresión concreta y material: la recaudación fiscal de un Estado, por ejemplo, permitiría tener una burocracia civil más eficiente, lo que a su vez permitiría obtener mayores ingresos (WALLERSTEIN, 2010, p. 500-501). Esto provoca que la fortaleza del centro y la debilidad de la periferia, se incrementen progresivamente en el propio ejercicio de la relación que desarrollan.

Bajo la lógica del sistema-mundo, esta relación asimétrica entre centro y periferia es necesaria para el funcionamiento de la economía-mundo capitalista. Si existiesen múltiples entidades políticas fuertes, incluyendo entes periféricos fuertes, entonces habría múltiples organismos en condiciones de perturbar y hasta bloquear el funcionamiento de las entidades económicas trasnacionales cuyo centro estuviese en otro Estado. Con esto, se perturbaría el funcionamiento de la división internacional del trabajo, y de esa forma la economía-mundo declinaría y el sistema podría venirse abajo (WALLERSTEIN, 2010, p. 499).

Más aún, la ausencia de un mecanismo político centralizado en la economía mundo hace difícil la introducción de fuerzas que sean capaces de contrarrestar este desequilibrio. De este modo, la debilidad estructural de las áreas periféricas se incrementa progresivamente sin un ente 
regulatorio en relación a la fuerza y autonomía de los Estados centrales (WALLERSTEIN, 2010).

Entre ambos extremos del eje se ubica la "semiperiferia", zonas "más ricas que la periferia" pero menos ricas que el centro, con desarrollos políticos, sociales, económicos y culturales intermedios (AGUIRRE, 2004, p. 43). Si bien, estas zonas tendrían mayor autonomía relativa que la periferia, al igual que esta última, se encuentran en una relación de subordinación respecto al centro.

Por fuera de los límites de la periferia, externa al sistema, se manifiesta la presencia de las "arenas exteriores". Áreas que el sistema explota y depreda, con la cual se establecen intercambios, pero que se mantiene fuera de sus fronteras. El sistema-mundo suele ir reduciendo estas "arenas exteriores" hasta incorporarlas definitivamente como periferia (AGUIRRE, 2004, p. 42).

La variable ambiental en el eje relacional centro-periferia. América Latina en su relación con los países centrales del sistema mundia

A inicios de la década de los ochenta, en el marco del proyecto de la CEPAL denominado Estilos de desarrollo y medio ambiente en la América Latina ${ }^{6}$, su director Osvaldo Sunkel y Luciano Tomassini (1980) planteaban que los factores ambientales emergían en el escenario mundial estrechamente vinculados a factores económicos, políticos y sociales, transformando el proceso de transnacionalización, el cual consideraban el rasgo dominante del sistema global contemporáneo, y con ello los estilos de desarrollo y la forma de participación internacional de los países periféricos.

En este marco, Sunkel y Tomassini (1980) establecían a lo menos tres factores. En primer lugar, las presiones sobre la capacidad de absorción del ecosistema. La cual en los países centrales se encontraba ya saturada sobre las últimas décadas del siglo XX, razón por la cual han debido reorientar sus actividades productivas contaminantes hacia los países periféricos, generando allí situaciones nocivas como la contaminación de mares y océanos, deforestación de selvas y bosques y generación de gases contaminantes hacia la atmósfera.

Si se considera la capacidad de absorción del ecosistema como un recurso económico, es posible concluir que aquella práctica de reorientación de las actividades productivas contaminantes hacia los países periféricos significa un uso abusivo por parte de los países industrializados de un "recurso relativamente escaso" del cual disponen los países de la periferia (SUNKEL; TOMASSINI, 1980, p. 304). Para los autores:

\footnotetext{
La progresiva limitación de esta capacidad de absorción a consecuencia de las presiones excesivas a que ha sido sometida por efecto del ritmo y forma que adoptó el crecimiento económico en los centros, tomará más agudo este problema y más valiosa la utilización de este recurso (SUNKEL; TOMASSINI, 1980, p. 304).
}

Un segundo factor se relaciona con la estrecha asociación entre el desarrollo económico y el uso de energía. En términos simples, los países centrales, altamente industrializados, denominados desarrollados, demandan y utilizan más energía que los países periféricos, primario exportadores, caracterizados como subdesarrollados o "en desarrollo". En
6. El proyecto Estilos de desarrollo y medio ambiente en la América Latina fue desarrollado en la CEPAL entre los

años 1978 y 1980 y "estuvo orientado al análisis de la relación del desarrollo con el medio ambiente" (GLIGO, 2006, p. 5). Para el Dr. Fernando Estenssoro (2014), este proyecto responde a los esfuerzos realizados desde hacía años atrás por la CEPAL y la Oficina Regional para América Latina y el Caribe (ORPALC) del Programa de Naciones Unidas para el Medio Ambiente (PNUMA) para "sensibilizar a los gobiernos de América Latina y el Caribe sobre la necesidad de introducir la variable ambiental en los temas del desarrollo", los cuales "en los primeros años no tuvieron buenos resultados" (Estenssoro, p. 144, 2014).

En el marco de este proyecto surgió, primero el seminario, y posteriormente la obra del mismo nombre Estilos de desarrollo y Medio Ambiente en la América Latina (1981), la cual: "se transformará en un impulso fundamental y clave para enfrentar la problemática ambiental en esta región del mundo" (ESTENSSORO, 2014, p. 147). Para Osvaldo Sunkel, director del proyecto, "a partir de las conceptualizaciones que hicimos con ese programa y del seminario, nos pasamos casi una década trabajando temas de medio ambiente y desarrollo. En los años siguientes se implementaron múltiples seminarios en (la) región con relación los temas que se tocaron en el libro" (SUNKEL apud ESTENSSORO, 2014, p. 147). 
7. Sunkel y Tomassini (1980) también plantean, aunque no exactamente de la misma forma y a partir de la misma reflexión, que los factores ambientales le otorga una mayor capacidad de negociación en el Sistema Internacional a la periferia. Lo cual, es una referencia importante para este trabajo, pero no es exactamente la misma reflexión. un grado relevante, los países centrales no logran satisfacer su demanda energética a partir de los recursos propios, pues, o bien no cuentan con la dotación suficiente para sus necesidades, dado su nivel de industrialización y/o alto estándar de vida, o simplemente ya la han agotado.

Este fenómeno revela una serie de situaciones problemáticas, entre ellas, por una parte la desigualdad en cuanto a los niveles de consumo de recursos naturales y energía entre centro y periferia, y por otro lado, que los "estilos de desarrollo" de los países centrales y las tecnologías que los sostienen "los han hecho extremadamente dependientes de una amplia disponibilidad de recursos naturales, que tardaron millones de años en crearse y cuyas reservas no son renovables" (SUNKEL; TOMASSINI, 1980, p. 304).

En tercer lugar, y en directa relación con los factores anteriores, siendo la capacidad de absorción del medio ambiente una condición necesaria para el desarrollo de ciertas actividades industriales, se genera progresivamente un movimiento de redistribución geográfica de aquellos procesos productivos, donde esa capacidad de absorción en combinación con la normativa ambiental, generan escenarios propicios desde el punto de vista y los intereses económicos del centro, para la instalación de determinadas actividades industriales en diferentes zonas de la periferia (SUNKEL; TOMASSINI, 1980, p. 311).

A partir de estos tres factores planteados por Sunkel y Tomassini (1980), buscando establecer elementos para responder directamente la pregunta que motiva este trabajo, es posible proponer que la CA aumenta el valor de recursos, elementos, bienes y servicios que están en la periferia, lo que por una parte, dadas las condiciones históricas de subordinación, provoca un aumento de las tasas de plusvalía en favor del centro, pero que sin embargo, y paradójicamente, le otorgaría a la periferia elementos para una potencial mayor capacidad de negociación con los países centrales ${ }^{7}$.

Esto se explica, pues, la CA, sus elementos fundamentales, principalmente sus efectos y consecuencias, generan modificaciones en las condiciones ecológicas del planeta, con lo cual se provoca una valorización o revalorización de determinados elementos y/o procesos ecológicos que están principalmente en las zonas periféricas, los cuales, algunos de ellos, se expresan en precios de mercado y son parte directa y explícitamente de las cadenas de valor, tales como recursos energéticos demandados por el centro para sus procesos productivos, zonas prístinas o al menos con capacidad de absorción y que potencialmente podrían ser objeto de localización de actividades productivas y contaminantes, recursos naturales de diverso tipo, biodiversidad, tanto como recurso en sí misma y como fuente de información y tecnología, y otros. Mientras, otros elementos y/o procesos ecológicos no se expresan en precios de mercado, pero sin embargo de igual forma están presentes en las cadenas de valor, pues sostienen el sistema y se hacen parte de las transferencias de excedentes de la periferia al centro, tales como la succión de gases de efecto invernadero, la generación de oxigeno desde zonas boscosas y selváticas, la preservación de biodiversidad e información genética desconocida, las reservas de agua dulce, y otros. Y en la medida en que ambos tipos, juntos o por separado, se valorizan o revalorizan, aumenta la tasa de transferencia de excedentes de la periferia al centro. 
La Iniciativa Yasuní ITT

Esta situación y estos elementos fueron puestos en tensión en la lógica y la problemática en torno a la propuesta denominada Iniciativa Yasuní ITT, la cual fue planteada internacionalmente por el Gobierno ecuatoriano en el año 2007 en Asamblea General de Naciones Unidas y liquidada por decreto en agosto de 2013 (VÁSQUEZ, 2015, p. 8).

Esta propuesta consistía en el compromiso de mantener bajo tierra indefinidamente 846 millones de barriles de reservas probadas de crudo al interior del Parque Nacional Yasuní, plena amazonia ecuatoriana, cantidad equivalente al $20 \%$ de la producción petrolera del país. Con lo cual, se evitaría la emisión de 407 millones de toneladas métricas de C02 producidas por la extracción y quema de aquellos combustibles fósiles, sumado a otros 800 millones de toneladas provocadas por la deforestación asociada a aquel procedimiento, considerando además un conjunto de impactos desencadenados tales como la migración de especies, la contaminación de aguas, la propagación de metano, y otros (REPÚBLICA DEL ECUADOR; PROGRAMA DE NACIONES UNIDAS PARA EL DESARROLLO, 2012; VÁSQUEZ, 2015).

Esta acción tendría lugar en uno de los territorios más biodiversos y ecológicamente sensibles del ecosistema terrestre, el Parque Nacional Yasuní (VÁSQUEZ, 2015). El cual, a su vez, es parte de una de las principales áreas boscosas del planeta, de las más grandes reservas de biodiversidad, información genética y oxigeno del mundo y "uno de los sumideros naturales de CO2 más importantes que existen...” como lo es la región amazónica (ESTENSSORO, 2010, p. 69). Es decir, un espacio natural que simultánea y permanentemente está resguardando la flora y fauna, emanando oxígeno y succionando gases contaminantes de la atmósfera.

Para dejar el petróleo bajo tierra, Ecuador le pedía a la comunidad internacional al menos $\$ 3.600$ millones de dólares en un plazo de 13 años, monto equivalente al $50 \%$ de lo que dejaría de percibir por efectuar esta acción. Los aportes podrían venir de Estados del mundo a través de sus gobiernos, la sociedad civil, empresas, o ciudadanos del planeta, sin embargo, se esperaba que las mayores contribuciones proviniesen de los países que se encuentran en el anexo I de la Convención Marco de Naciones Unidas sobre el Cambio Climático, es decir, la lista de países industrializados más contaminantes del globo (REPÚBLICA DEL ECUADOR; PROGRAMA DE NACIONES UNIDAS PARA EL DESARROLLO, 2012; VÁSQUEZ, 2015).

Tras décadas de DA, y en un contexto de agudización de los desequilibrios ecológicos, esta Iniciativa se constituyó en una propuesta concreta en el escenario internacional que permitiría contribuir a la mitigación del calentamiento global y al combate contra la pérdida de biodiversidad, dos de los principales efectos de la CA en directa relación con el cambio climático, y que abordaba estas problemáticas desde la realidad de un país periférico y poniendo en tensión, desde su perspectiva, las propias asimetrías entre países del centro y la periferia (VÁSQUEZ, 2015, p. 9; ESTENSSORO; VÁSQUEZ, 2017).

Así, cuando Ecuador planteaba una compensación del 50\% de lo que dejaría de percibir por mantener el petróleo bajo tierra, y que esta 
debía venir de la comunidad internacional, estaba hablando de CORRESPONSABILIDAD: Todos quienes habían sido parte de la generación de la CA debían hacerse cargo de ella. Sin embargo, al proponer que ese dinero debía venir principalmente de los países industrializados, hablaba de CORRESPONSABILIDAD DIFERENCIADA (VÁSQUEZ, 2015). Es decir, estaba proponiendo la aplicación del principio de las Responsabilidades Comunes pero Diferenciadas, el cual parte del reconocimiento de que no son todos los países son responsables por igual frene a la CA.

Pues, en un mundo desigual, existe una relación directamente proporcional en cuanto a nivel de ingreso y consumo de ambiente, es decir, contaminación ambiental. Los países que más contaminan son los más ricos, mientras que, quienes menos contaminan son los más pobres. Relación asimétrica de más de cien veces a uno (CORREA, 2012). Sin embargo, los países pobres y subdesarrollados, como los de la cuenca amazónica, son los mayores proveedores de aire puro del mundo. Es decir, quienes generan bienes y valores ambientales son los que menos los consumen, y quienes más los consumen son quienes no los producen (VÁSQUEZ, 2015).

La propuesta planteada mediante esta iniciativa permitía, si no bien controlar, a lo menos intervenir o llamar la atención respecto a ese aumento de plusvalía desde la periferia al centro a partir de las modificaciones que genera la CA. Pues, está explícitamente planteando un precio por dejar el petróleo bajo tierra, y con ello, por el valor de la generación de oxígeno, la absorción de gases contaminantes de efecto invernadero, y el cuidado y mantenimiento de la biodiversidad y las zonas no transformadas por los procesos productivos. Es decir, esta iniciativa proponía una fórmula para cobrar por un servicio que ya se está prestando y por el cual no se está pagando. Si bien, ese territorio amazónico está dentro de la jurisdicción ecuatoriana, esa generación de oxígeno y esa succión de gases contaminantes de efecto invernadero tiene consecuencias directas globales.

Además de lo anterior, esta iniciativa involucra una nueva forma de valorización del petróleo a partir de un uso diferente. Pues, bajo esta lógica, el petróleo no deja de ser un recurso económico, lo que plantea es modificar el medio de cambio, uso y compensación, al reconocer el valor global de la generación de oxígeno, la succión de gases contaminantes y la preservación de la biodiversidad, y establecer un precio por aquello. Es decir, esta iniciativa no excluye el petróleo ni de los mercados ni de las cadenas de producción ni valor, sino que, a través de la omisión de una acción, su extracción, a la que Ecuador tiene derechos soberanos, se le hace parte de aquel proceso de generación de valor, usos y servicios (VÁSQUEZ, 2015, p. 10-11).

Situación que, por lo demás, al tratarse del petróleo, agudiza la hipotética tensión. Como lo plantearan Sunkel y Tomassini (1980, p. 305), hay una estrecha relación entre consumo de energía y desarrollo económico, particularmente en cuanto a los estilos de desarrollo prevalecientes en los países centrales. Es decir, la Iniciativa Yasuní ITT planteaba una intervención en los usos del recurso energético que ha movilizado los sistemas productivos en el último siglo y medio, y que está presente en los estándares de vida y la vida cotidiana de la sociedad, se trata de la "sangre del capitalismo industrial". Intervenir sus procesos implica arriesgar una 
baja de su producción, alterar sus precios, modificar encadenadamente otros precios de mercado, ralentizar los ritmos de acumulación, entre otros posibles efectos.

Finalmente, tras seis años de promoción y negociaciones, en agosto de 2013, el Gobierno ecuatoriano firma el decreto que liquidaba la iniciativa Yasuní ITT, argumentando principalmente que la comunidad internacional no había respondido a la propuesta, pues los aportes esperados no se habían recaudado, y que el país necesitaba utilizar los potenciales recursos económicos provenientes del petróleo, hasta ese momento bajo tierra, en función de financiar políticas sociales y de desarrollo (CORREA, 2013, p. 16).

Efectivamente, de los 3.600 millones de dólares esperados, en agosto de 2013 se habían reunido 13,3 millones, un 0,37\% del monto planteado como objetivo inicial (CORREA, 2013). Entre los contribuyentes se encontraban solamente 8 gobiernos de estados y 4 gobiernos regionales de otros dos países (PROGRAMA DE NACIONES UNIDAS PARA EL DESARROLLO, 2014). De aquellas diez naciones apenas 5 se encuentran en el Anexo I de la Convención Marco de Naciones Unidas sobre el Cambio Climático, y solamente uno de ellos está dentro de los cinco primeros países de esta lista ${ }^{8}$, es decir, el grupo de países más contaminantes y los mayores consumidores de recursos naturales, y que a la vez son las economías centrales más grandes y desarrolladas.

Es decir, no solamente los dineros no se recaudaron, significando el fracaso concreto y mecánico de la Iniciativa Yasuní ITT, sino que además las potencias centrales de la economía mundo al mantenerse al margen, negarse a contribuir, o sencillamente omitir pronunciamientos, rechazaron la propuesta.

Es complejo abordar en su totalidad las razones de este fracaso, sin embargo, en el ámbito que interesa a este trabajo, las palabras del Ministro de Desarrollo alemán en el año 2011, Dirk Niebel, quien rechazó el proyecto ecuatoriano y criticó duramente el apoyo de Italia al programa, son expresivas de aquellas causas, al afirmar que si apoyaran la propuesta ecuatoriana estarían sentando un precedente con "derivaciones imprevisibles" (EL COMERCIO..., 2011).

En ese mismo sentido, como plantearan Estenssoro y Vásquez (2017):

¿Qué pasaría en el orden internacional económico y geopolítico, si países exportadores de gas, uranio, cobre $\mathrm{u}$ otro recurso natural solicitan al Primer Mundo que se les pague -un precio justo o de acuerdo a lo que dejarían de ganar-, por no explotar estos recursos, a fin de mitigar las externalidades ambientales negativas que estas actividades conllevan? ¿Qué pasaría con el macro sistema económico-industrial y financiero del centro que necesita de manera creciente estos recursos y al más bajo precio posible, tanto para seguir funcionando, como para seguir beneficiándose de las relaciones de intercambio económico internacional? (ESTENSSORO; VÁSQUEZ, 2017, p. 76).

Conclusión

El ejercicio académico y científico de vincular la CA como variable en el eje relacional centro periferia según la perspectiva teórica AS-M, es una posibilidad abierta y necesaria en función de un mayor entendimiento del desarrollo del capitalismo histórico y el lugar y rol de América Latina en ello.
8. Hasta agosto de 2013, los gobiernos contribuyentes fueron: Italia, con 3.932.147 dólares; España, con 1.400.400 dólares; Luxemburgo, con 1.332.700 dólares; Chile, con 100.000 dólares, Georgia, con 100.000 dólares, Colombia, con 100.000 dólares; y Turquía, con 100.000 dólares. Los gobiernos regionales de: Región de Valonia, Bélgica, con 1.220 .465 dólares; Region Rhone-Alpes, Francia, con 195,314 dólares; Región Meurthe-et-Moselle, Francia, 53,763 dólares; Región Limousin, Francia, con un aporte inferior a 50.000 dólares. 
La CA introduce elementos de modificación y cambio en aquella relación. No ha invertido los roles ni equilibrado las relaciones asimétricas entre centro y periferia, sin embargo, abre escenarios críticos para la economía mundo y espacios de oportunidad. Por una parte, plantea límites a las lógicas y dinámicas de acumulación capitalista, lo que a su vez podría empujar la creación de formas y mecanismos que le permitan superar esos límites, por otro lado revaloriza determinados elementos y procesos del ecosistema, lo que aumenta las tasas de transferencia de excedentes de la periferia al centro, pero que a su vez, le otorgaría un mayor carácter estratégico a esos procesos, elementos y recursos, y por tanto podría brindar mayores herramientas y potenciales oportunidades en favor de la periferia.

Desde su origen, la relación entre la economía-mundo capitalista y los procesos ecológicos es vital, la transformación/depredación ambiental es efecto y a la vez motor, elemento movilizador de la expansión del capitalismo. En este sentido las modificaciones en los procesos y elementos del ecosistema, los efectos de la CA, son variables en la evolución, desarrollo y posibles cambios en la economía mundo capitalista y en el eje relacional sobre el cual se organiza su división del trabajo.

Referencias

AGUIRRE, C. Immanuel Wallerstein: crítica del sistema-mundo capitalista. Estudio y entrevista. Santiago: LOM, 2004

CORREA, R. La Iniciativa Yasuní-ITT: Cambiando paradigmas para un futuro sustentable. 20 jun. 2012. Disponível em: <http://www.presidencia.gob.ec/wp-content/uploads/ downloads/2012/10/2012-06-20-Iniciativa-Yasuní-ITT-Cambiando-paradigmas-para-un-futuro-sustentable.pdf $>$ Acesso em: 20 out. 2017.

CORREA, R. Anuncio a la Nación Iniciativa Yasuní ITT. 15 ago. 2013. Disponível em: <https://www.presidencia.gob.ec/wp-content/uploads/downloads/2013/08/2013-08-15 AnuncioYasuni.pdf $>$. Acesso em: 20 out. 2017.

EL COMERCIO. Duras críticas de Alemania a proyecto ecuatoriano de Yasuní-ITT". El Comercio. 9 out. 2011. Disponível em: <http://www.elcomercio.com/tendencias/duras-criticas-de-alemania-a.html>. Acesso em: 04 jan 2016.

ESTENSSORO, F. Antecedentes para una historia del debate político en torno al medio ambiente: la primera socialización de la idea de crisis ambiental(1945 - 1972). Revista UNIVERSUM, Talca, vol. 22, n. 2, p. 88-107, 2007.

ESTENSSORO, F. Medioambiente e ideología. La discusión pública en Chile, 1992- 2002. Antecedentes para una historia de ideas políticas a inicios del siglo XXI. Santiago: Ariadna, 2009.

ESTENSSORO, F. Crisis ambiental y cambio climático en la política global: un tema crecientemente complejo para América Latina. Revista UNIVERSUM, Talca, vol. 2, n. 25, p. 57-77, 2010.

ESTENSSORO, F. Historia del debate ambiental en la política mundial 1945-1992. La perspectiva latinoamericana. Santiago: Instituto de Estudios Avanzados, Universidad de Santiago de Chile, 2014.

ESTENSSORO, F; VÁSQUEZ, J. Las diferencias Norte-Sur en el Debate Ambiental global. El caso de la propuesta del Ecuador: Yasuní - ITT. Revista UNIVERSUM, Talca, vol. 32, n. 2, p. 63-80, 2017.

FOSTER, J. B. Marx's theory of metabolic rift: Classical foundations for environmental sociology. American Journal of Sociology, vol. 105, n. 2, p. 366-405, 1999.

GLIGO, N. Estilos de desarrollo y medio ambiente en la América Latina, un cuarto de siglo después. Serie Medio ambiente y desarrollo. Santiago: CEPAL, 2006.

LE QUANG, M. Y VERCOUTERE, T. Ecosocialismo y Buen Vivir. Diálogo entre dos alternativas al capitalismo. Quito: Editorial IAEN, 2015. 
MOORE, J. El auge de la economía mundo capitalista I. Las fronteras mercantiles en el auge y decadencia de la apropiación máxima. Laberinto, $n$. 38, 9-26, 2013b. Disponível em: < http:// laberinto.uma.es/index.php?option=com_content $\&$ view $=$ article $\&$ id $=574$ :el-auge-de-la-ecologia-mundo-capitalista-i-las-fronteras-mercantiles-en-el-auge-y-decadencia-de-la-apropiacion-maxima\&catid=128:lab38\&Itemid=54>. Acesso em: 14 abr. 2018.

MOORE, J. El auge de la economía mundo capitalista II. Las fronteras mercantiles en el auge y decadencia de la apropiación máxima. Laberinto, n. 39, 21-29, 2013a. Disponível em: <http://laberinto.uma.es/index.php?option $=$ com_content $\&$ view $=$ article $\&$ id $=585$ :el-auge-de-la-ecologia-mundo-capitalista-ii-las-fronteras-mercantiles-en-el-auge-y-decadencia-de-la-apropiacion-maxima\&catid=130:laberinto-39\&Itemid=54>. Acesso em: 14 abr. 2018

MOORE, J. The Modern World-System as environmental history? Ecology and the rise of capitalism. Theory and Society, vol. 32, n. 3, p. 307-377, 2003a.

MOORE, J. Capitalism as World-Ecology. Braudel and Marx on Environmental History. Organization \& Environment, vol. 16, n. 4, 431-458, 2003b.

ORTIZ, E. EI estudio de las relaciones internacionales. Santiago: Fondo de Cultura Económica, 2011.

PROGRAMA DE NACIONES UNIDAS PARA EL DESARROLLO. Ecuador Yasuni Capital Window. Financial Reporting on Sources and uses of Found. For the period ending 31 de diciembre 2014. 2014. Disponível em: <http://mptf.undp.org/factsheet/fund/3EYC0/es >. Acesso em: 5 jan. 2016.

REPÚBLICA DEL ECUADOR; PROGRAMA DE NACIONES UNIDAS PARA EL DESARROLLO. Ecuador Yasuni ITT Initiative Fact Sheet. 2012. Disponível em: <http://mptf.undp. org/yasuni>. Acesso em: 5 jan. 2016.

SUNKEL, O; TOMASSINI, L. Los factores ambientales y el cambio en las relaciones internacionales de los países en desarrollo. En: Sunkel O; Gligo N. Estilos de desarrollo y Medio Ambiente. México: Fondo de Cultura Económica, cap 8, p. 288-319. 1980.

VÁSQUEZ, J. P. La tensión histórica norte - sur global en el debate ambiental. El conflicto en torno a la iniciativa Yasuní ITT. Revista Estudios Hemisféricos y Polares, v. 6, n. 1, p. 1-28, 2015.

VÁSQUEZ, J. P. Medioambiente, Desarrollo y Soberanía: La Tensión Norte sur Global en el Debate Medioambiental. El Caso de la Iniciativa Yasuní-ITT del Estado Ecuatoriano como Propuesta de Política Pública Global desde América Latina. 2014. Tesis, Magíster en Estudios Internacionales, Instituto de Estudios Avanzados, Universidad de Santiago de Chile.

WALLERSTEIN, I. La Ecología y los costos de producción capitalistas. No hay salida. En: Wallerstein, I. Conocer el mundo, saber el mundo: el fin de lo aprendido. Una ciencia social para el siglo XXI. México D. F.: Siglo XXI, cap 5, p. 88-99. 2001a.

WALLERSTEIN, I. ¿Estados? ¿Soberanía? Los dilemas de los capitalistas en una época de transición. En: Wallerstein, I. Conocer el mundo, saber el mundo: el fin de lo aprendido. Una ciencia social para el siglo XXI. México D. F.: Siglo XXI, cap 4, p. 67-87. 2001b.

WALLERSTEIN, I. Análisis de sistemas-mundo. Una introducción. México D. F.: Siglo XXI, 2005.

WALLERSTEIN, I. Impensar las Ciencias Sociales. Límites de los paradigmas decimonónicos. México D. F.: Siglo XXI, 2007a.

WALLERSTEIN, I. La decadencia del imperio. Estados Unidos en un mundo caótico. Caracas: Monteavila Editores Latinoamericana, 2007b.

WALLERSTEIN, I. El moderno sistema mundial I. La agricultura capitalista y los orígenes de la economía-mundo europea en el siglo XVI. Madrid: Siglo XXI, 2010. 